\title{
Matrix-assisted laser desorption ionization time-of-flight mass spectrometry for rapid identification of mold and yeast cultures of Penicillium marneffei
}

Susanna K. P. Lau ${ }^{1,2,3,4^{*}+}$, Clare S. K. Lam ${ }^{5 \dagger}$, Antonio H. Y. Ngan ${ }^{4}$, Wang-Ngai Chow ${ }^{4}$, Alan K. L. Wu $u^{6}$, Dominic N. C. Tsang ${ }^{7}$, Cindy W. S. Tse ${ }^{8}$, Tak-Lun Que ${ }^{9}$, Bone S. F. Tang ${ }^{5}$ and Patrick C. Y. Woo 1,2,3,4*

\begin{abstract}
Background: Penicillium marneffei is the most important thermal dimorphic fungus causing systemic mycosis in HIV-infected and other immunocompromised patients in Southeast Asia. However, laboratory diagnosis of penicilliosis, which relies on microscopic morphology and mycelial-to-yeast conversion, is time-consuming and expertise-dependent, thus delaying diagnosis and treatment. Although matrix -assisted laser desorption ionization time-of-flight mass spectrometry (MALDI-TOF MS) is useful for identification of various medically important fungi, its performance for identification of $P$. marneffei is less clear.

Results: We evaluated the performance of the Bruker MALDI-TOF MS system for identification of mold and yeast cultures of 59 clinical strains and the type strain of $P$. marneffei using the direct transfer method, with results compared to four phylogenetically closely related species, P. brevi-compactum, P. chrysogenum, Talaromyces aurantiacus and T. stipitatus. Using the Bruker original database combined with BDAL v4.0.0.1 and Filamentous Fungi Library 1.0, MALDI-TOF MS failed to identify the $60 \mathrm{P}$. marneffei strains grown in mold and yeast phase (identified as $P$. funiculosum and $P$. purpurogenum with scores $<1.7$ respectively). However, when the combined database was expanded with inclusion of spectra from $21 P$. marneffei strains in mold and/or yeast phase, all the remaining $39 P$. marneffei strains grown in mold or phase were correctly identified to the species level with score $>2.0$. The MS spectra of $P$. marneffei exhibited significant difference to those of $P$. brevi-compactum, $P$. chrysogenum, $T$. aurantiacus and $T$. stipitatus. However, MALDI-TOF MS failed to identify these four fungi to the species level using the combined database with or without spectra from $P$. marneffei.
\end{abstract}

Conclusions: MALDI-TOF MS is useful for rapid identification of both yeast and mold cultures of $P$. marneffei and differentiation from related species. However, accurate identification to the species level requires database expansion using P. marneffei strains.

Keywords: MALDI-TOF, MS, Rapid, Identification, Pencillium, marneffei

\footnotetext{
* Correspondence: skplau@hkucc.hku.hk; pcywoo@hkucc.hku.hk

${ }^{\dagger}$ Equal contributors

'State Key Laboratory of Emerging Infectious Diseases, The University of Hong Kong, Hong Kong, China

${ }^{2}$ Research Centre of Infection and Immunology, The University of Hong

Kong, University Pathology Building, Queen Mary Hospital, Hong Kong, China

Full list of author information is available at the end of the article
} 


\section{Background}

Penicillium marneffei is the most important thermal dimorphic fungus causing respiratory, skin and systemic mycosis in Southeast Asia [1-4]. After its first discovery in bamboo rats [5, 6], only 18 cases of human diseases were reported until 1985 [7]. The HIV pandemic in the 1980's has resulted in a surge of HIV-associated P. mar$n e f f e i$ infections in Southeast Asia where the fungus is endemic [2]. In Hong Kong, about $10 \%$ of HIV patients have been infected with $P$. marneffei $[8,9]$. In addition to HIV-infected patients, penicilliosis is also an emerging disease in other immunocompromised patients such as transplant recipients and patients on immunosuppressant therapies [10-13]. Imported cases of $P$. marneffei infections have also been reported in nonendemic countries [14, 15]. Despite its medical importance, the mode of transmission, and dimorphic and pathogenic mechanisms of $P$. marneffei remain poorly understood [16-18].

Diagnosis of penicilliosis can be difficult, since many clinical laboratories are inexperienced in identifying this dimorphic fungus. P. marneffei exhibits distinct cellular morphologies at different temperatures, in mycelial phase at $25{ }^{\circ} \mathrm{C}$ and yeast phase at $37{ }^{\circ} \mathrm{C}$ [19]. Laboratory diagnosis relies on either direct examination of infected tissues that contain $P$. marneffe $i$ yeast-like cells or, more commonly, positive cultures from tissues or blood. Although the production of blue-green to yellowish colonies from cultures at $25{ }^{\circ} \mathrm{C}$ with a soluble diffusible red pigment on agar is highly suggestive of $P$. marneffei, other Penicillium or related species can also produce similar pigments [20]. Therefore, definitive identification of $P$. marneffei often requires the demonstration of mycelial-to-yeast conversion, which typically shows oval, yeast-like cells with abortive, branched and separate hyphae at $37{ }^{\circ} \mathrm{C}$, and. However, the entire process takes approximately two weeks As a result, the identification of P. marnef$f e i$ is often time-consuming and requires considerable experience, which may lead to delayed diagnosis and treatment.

Conventional phenotypic methods and commercial kits may not readily identify the less commonly encountered dimorphic fungi. On the other hand, sequencing of conserved gene targets for fungal species identification is expensive and requires special expertise. Matrix-assisted laser desorption ionization time-of-flight mass spectrometry (MALDI-TOF MS) has recently emerged as a revolutionary technique for pathogen identification, yielding rapid, accurate and highly reproducible results at a lower price than any other routine methods used in clinical microbiology laboratories, although the equipment itself requires substantial initial investment [21-23]. The methodology is easy to follow even with an inexperienced operator, and the results are available within minutes. Therefore, the technique is now integrated into many clinical laboratories. MALDITOF MS is useful for identification of various bacterial pathogens, including those less common species [24-26]. Recent studies have shown that MALDI-TOF MS is also capable of identifying various medically important fungi, including both yeasts, e.g. Candida and Cryptococcus, and molds, e.g. Aspergillus, Fusarium and dermatophytes [27-36]. The technique has also been extended to the development of antifungal susceptibility tests for Candida and Aspergillus [37, 38]. Since only minimal amounts of micro-organisms are required, the technique is also potentially advantageous over conventional identification for "dangerous" pathogens, such as Burkholderia pseudomallei and dimorphic fungi, which require biosafety level 3 laboratories for culture [25]. However, there has been no study focusing on the usefulness of MALDI-TOF MS for identification of dimorphic fungi such as $P$. marneffei. In this study, we evaluated the performance of MALDI-TOF MS in identifying both the mold and yeast cultures of 60 P. marneffei strains.

\section{Methods \\ Fungal strains and culture conditions}

A total of $60 P$. marneffei strains were included, including 59 clinical strains isolated from patients with culture-documented penicilliosis and P. marneffei type strain ATCC $18224^{\mathrm{T}}$ isolates from a bamboo rat [39]. All the $59 \mathrm{P}$. marneffei clinical strains were identified by sequencing of three housekeeping genes, mannose phosphate isomerase (MPI), plasma membrane $\mathrm{H}+$ ATPase (PM-ATPase) and pyruvate kinase (PK), as described previously [40]. The sequences of all three housekeeping genes were identical among the 59 clinical strains and $P$. marneffei type strain ATCC $18224^{\mathrm{T}}$ (data not shown). Since P. marneffei is phylogenetically closely related to other Penicillium species and Talaromyces which is the telemorph of some Penicillium species [18, 41], two strains of other Penicillium species (P. brevi-compactum ATCC 14586 and P. chrysogenum ATCC 9480) and two strains of Talaromyces species (Talaromyces aurantiacus strain PW3105 and T. stipitatus ATCC 10500) were also included. T. aurantiacus strain PW3105 was isolated from the bronchial trap of a patient with left lung mass. All P. marneffei strains were grown on Sabouraud dextrose agar (SDA) (Oxoid, Cambridge, UK) at $37{ }^{\circ} \mathrm{C}$ for yeast cultures and at $25{ }^{\circ} \mathrm{C}$ for mold cultures for 4 to 7 days as described previously $[17,18]$. Yeast and mold forms of $P$. marneffei were collected by scraping and resuspension in $300 \mu \mathrm{l}$ sterile water. The four strains, $P$. brevi-compactum, $P$. chrysogenum, $T$. aurantiacus and $T$. stipitatus were grown on SDA at $25^{\circ} \mathrm{C}$ for 3 to 5 days. 


\section{MALDI-TOF MS}

All isolates were tested in duplicates by MALDI-TOF MS using ethanol-formic acid extraction protocol according to the manufacturers' instructions. Both yeast and mold cultures of $P$. marneffei, and mold cultures of P. brevi-compactum, $P$. chrysogenum, T. aurantiacus and $T$. stipitatus, were analyzed by the direct transfer method using the same experimental conditions [25, 42]. Briefly, single colonies were washed in $300 \mu \mathrm{l}$ sterile $\mathrm{H}_{2} \mathrm{O}$ and then resuspended in $900 \mu \mathrm{l}$ absolute ethanol followed by centrifugation at $13,000 \times \mathrm{g}$ for $2 \mathrm{~min}$. The supernatant was then removed and the pellet was allowed to air dry for $5 \mathrm{~min}$. The pellet was mixed with $50 \mu \mathrm{l} 70 \%$ formic acid by vortex. Fifty microliters of $100 \%$ acetonitrile were added to the solution and was mixed by pipetting. After centrifugation at $13,000 \times \mathrm{g}$ for $2 \mathrm{~min}, 1 \mu \mathrm{l}$ of the supernatant was transferred onto a spot of a polished steel target plate in a thin film (Bruker Daltonik, Bremen, Germany). Each spot was then airdried and overlaid with $1 \mu \mathrm{l}$ matrix solution ( $\alpha$-cyano-4hydroxycinnamic acid in $50 \%$ acetonitrile and $2.5 \%$ trifluoroacetic acid) (Bruker Daltonik). After crystallization of the matrix solution, the target was loaded into the MALDI-TOF MS spectrometer (Bruker Daltonik) for analysis. Spectra were obtained with an accelerating voltage of $20 \mathrm{kV}$ in linear mode and analyzed within an $\mathrm{m} / \mathrm{z}$ charge of 2,000 to $20,000 \mathrm{Da}$. Spectra were analyzed with MALDI Biotyper $^{\mathrm{Tm}} 3.0$ software against the combined database with Reference Library BDAL v4.0.0.1 (Bruker Daltonik) and Filamentous Fungi Library 1.0 (Bruker Daltonik), with or without inclusion of additional spectra from $P$. marneffe $i$ strains grown in yeast or mold phase. Since $P$. marneffei is not represented in the Bruker Daltonik database, both yeast and mold cultures of 20 P. marneffei strains were later added as reference strains (Table 1). The MALDI Biotyper output is a log (score) in the range 0 to 3.0. Thresholds for species and genus identification were $\geq 2.0$ and $\geq 1.7$ respectively. If the scores from the first run were $<2.0$, a second run in duplicate was immediately performed. The highest of all the scores was considered the final result. Results are presented with the species or genus identification (above the cutoff scores). Scores below the cutoff were considered invalid results with the conclusion "no identification". Bruker bacterial test standard (BTS, no. 255343, Bruker Daltonics) was used for calibration and quality control in each run. Obtained spectra were subject to hierarchical cluster analysis using ClinProTools 3.0 (Bruker Daltonics) as described previously [43].

\section{Results}

Representative spectra obtained with mold and yeast cultures of $P$. marneffei compared to those obtained with mold cultures of $P$. brevi-compactum, $P$. chrysogenum, $T$. aurantiacus and T. stipitatus are shown in Fig. 1. They exhibited significant difference between the five different fungal species, suggesting that MALDI-TOF MS is potentially useful to differentiate between $P$. marneffei and related fungal species. In contrast, the spectra of the mold and yeast cultures of $P$. marneffei are similar. The MALDI-TOF MS identification results of the $60 P$. marneffei strains are represented in Table 1 . Using the

Table 1 Comparison of identification results by Bruker database and expanded database with $P$. marneffei strains

\begin{tabular}{|c|c|c|c|c|}
\hline Database & Fungi (no. of strains) & Growth form & Top rank identification & Logscore range \\
\hline Bruker database & Penicillium marneffei (60) & Mold & Penicillium funiculosum & $1.207-1.554$ \\
\hline [include BDAL (Bruker, Version 4.0.0.1), & Penicillium marneffei (60) & Yeast & Penicillium purpurogenum & $1.277-1.566$ \\
\hline \multirow[t]{4}{*}{ Filamentous Fungi Library 1.0 (Bruker)] } & Penicillium brevi-compactum (1) & Mold & Penicillium brevi-compactum & 1.736 \\
\hline & Penicillium chrysogenum (1) & Mold & Penicillium chrysogenum & 1.689 \\
\hline & Talaromyces aurantiacus (1) & Mold & Penicillium purpurogenum & 1.499 \\
\hline & Talaromyces stipitatus (1) & Mold & Pseudomonas putida & 1.343 \\
\hline \multirow{6}{*}{$\begin{array}{l}\text { Expanded Bruker database with inclusion } \\
\text { of spectra from } 20 \text { P. marneffei strains } \\
\text { grown in mold form }\end{array}$} & Penicillium marneffei (40) & Mold & Penicillium marneffei & $2.355-2.631$ \\
\hline & Penicillium marneffei (40) & Yeast & Penicillium marneffei & $2.130-2.607$ \\
\hline & Penicillium brevi-compactum (1) & Mold & Penicillium brevi-compactum & 1.714 \\
\hline & Penicillium chrysogenum (1) & Mold & Penicillium chrysogenum & 1.689 \\
\hline & Talaromyces aurantiacus (1) & Mold & Penicillium marneffei & 1.604 \\
\hline & Talaromyces stipitatus (1) & Mold & Lactobacillus plantarum & 1.368 \\
\hline \multirow{2}{*}{$\begin{array}{l}\text { Expanded Bruker database with inclusion } \\
\text { of spectra from } 20 \text { P. marneffei strains grown } \\
\text { in yeast form }\end{array}$} & Penicillium marneffei (40) & Mold & Penicillium marneffei & $2.150-2.575$ \\
\hline & Penicillium marneffei (40) & Yeast & Penicillium marneffei & $2.264-2.777$ \\
\hline \multirow{2}{*}{$\begin{array}{l}\text { Expanded Bruker database with inclusion of } \\
\text { spectra from } 20 \text { P. marneffei strains grown in } \\
\text { both mold and yeast form }\end{array}$} & Penicillium marneffei (40) & Mold & Penicillium marneffei & $2.396-2.628$ \\
\hline & Penicillium marneffei (40) & Yeast & Penicillium marneffei & $2.389-2.689$ \\
\hline
\end{tabular}




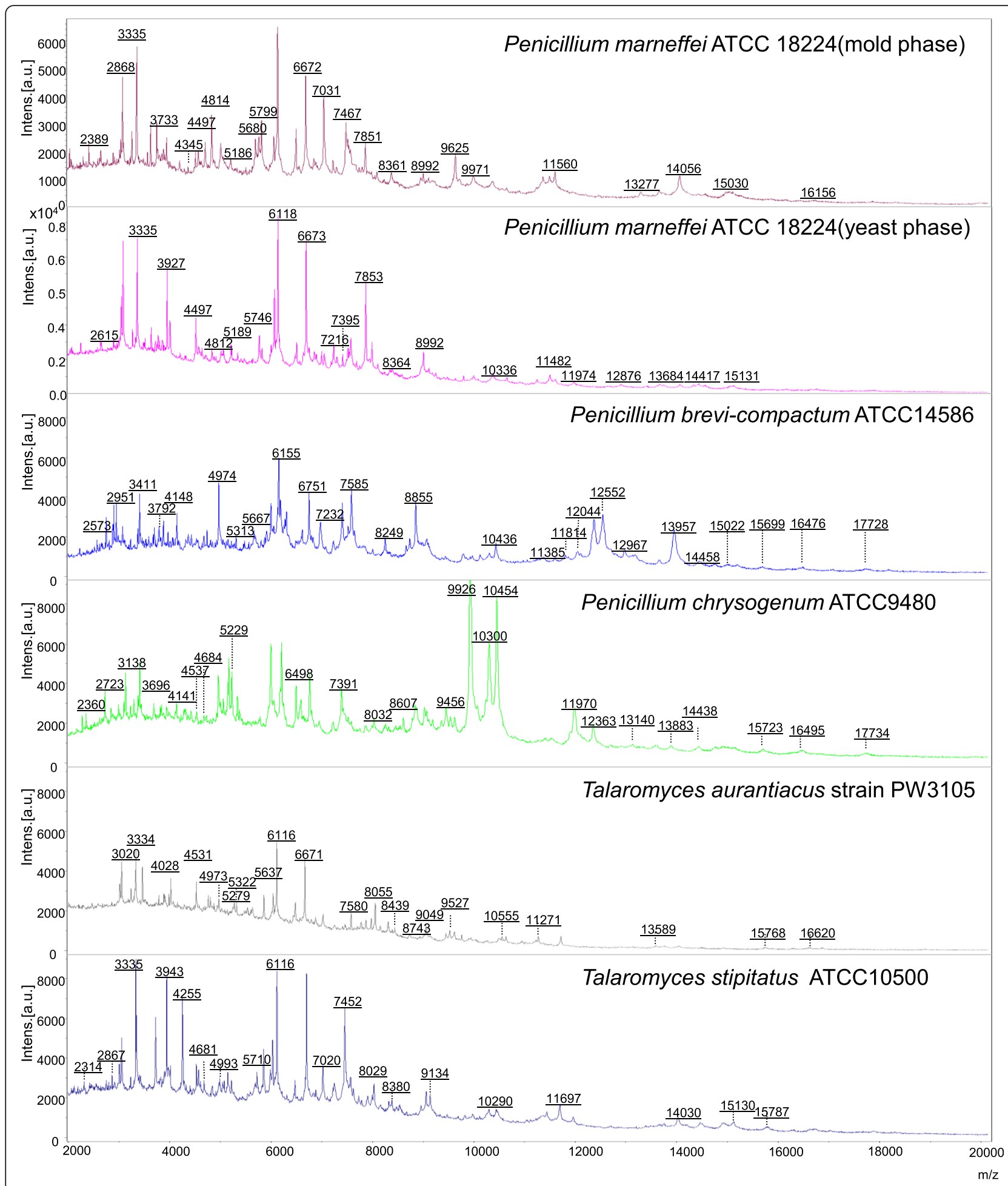

Fig. 1 MALDI-TOF mass spectra of mold and yeast cultures of $P$. marneffei compared to those of $P$. brevi-compactum, $P$. chrysogenum, $T$. aurantiacus and T. stipitatus.

combined database with BDAL v4.0.0.1 (Bruker Daltonik) and Filamentous Fungi Library 1.0 (Bruker Daltonik), without inclusion of additional spectra from $P$. marneffei strains, the $60 P$. marneffei strains grown in mold phase were identified as $P$. funiculosum with score 1.207-1.554 (indicating no identification); while these 60 strains grown in yeast phase were identified as $P$. purpurogenum with score 1.277-1.566 (indicating no 
identification). P. brevi-compactum and $P$. chrysogenum were correctly identified to the species level but only with score 1.735 and 1.689 respectively (indicating genus level and no identification respectively). T. aurantiacus and T. stipitatus were identified as P. purpurogenum and Pseudomonas putida with score 1.499 and 1.343 respectively (indicating no identification).

When the above combined database was expanded with inclusion of spectra from $21 \mathrm{P}$. marneffei strains (including 20 clinical strains and type strain $18224^{\mathrm{T}}$ ) grown in mold phase, all the remaining 39 P. marneffei strains grown in both mold and yeast phase were correctly identified to the species level with score 2.3552.631 and $2.130-2.607$ respectively. P. brevi-compactum and $P$. chrysogenum were again identified to the species level but with score 1.714 and 1.689 respectively (indicating genus level and no identification respectively). $T$. aurantiacus and $T$. stipitatus were identified as $P$. marneffei and Lactobacillus plantarum with score 1.604 and 1.368 respectively (indicating no identification).

When the combined database was expanded with inclusion of spectra from the $21 P$. marneffei strains grown in yeast phase, all the remaining $39 P$. marneffei strains grown in both mold and yeast phase were also correctly identified to the species level with score $>2.0$. Similarly, when the combined database was expanded with inclusion of spectra from $21 P$. marneffei strains grown in both mold and yeast phase, all the remaining $39 P$. marneffei strains grown in both mold and yeast phase were correctly identified to the species level with score $>2.0$. Hierarchical cluster analysis showed that the protein mass spectra of mold and yeast forms of $P$. marneffei were clustered together but formed a distinct branch being most closely related to P. funiculosum (Table 2, Fig. 2).

\section{Discussion}

Despite the usefulness of MALDI-TOF MS for various fungi as demonstrated by many studies [27-35], its performance on dimorphic fungi is less well understood. In one study in which the MALDI-TOF MS database was developed using 294 isolates of various molds, only 10 strains of thermally dimorphic fungi (four Histoplasma capsulatum two Coccidioides immitis, one each of Blastomyces dermatitidis, Paracoccidioides brasiliensis, P. marneffei and Sporothrix schenkii) were included [44]. Although $H$. capsulatum isolates were identified successfully, other dimorphic fungal species including $P$. marneffei were not included for evaluation [44]. In this study, all the $60 P$. marneffei strains failed to be identified using the combined Bruker and in-house database alone. The best matches were $P$. funiculosum and $P$. purpurogenum for mold and yeast cultures respectively with score $<1.7$, indicating "no identification". Nevertheless, fungal isolates, especially blood culture isolates from
Table 2 Spectra of Penicillium strains used for hierarchical cluster analysis

\begin{tabular}{lll}
\hline Species & Strain & Source \\
\hline Penicillium dierckxii & DSM 62842 & Bruker database \\
Penicillium citrinum & DSM 1179 & Bruker database \\
Penicillium discolor & MPA 1338 & Bruker database \\
Penicillium sp & MPA 1326 & Bruker database \\
Penicillium daleae & UGB 743 & Bruker database \\
Penicillium corylophilum & MPA 1111001 & Bruker database \\
Penicillium glabrum & DSM 16516 & Bruker database \\
Penicillium striatisporum & DSM 2439 & Bruker database \\
Penicillium citreonigrum & DSM 2427 & Bruker database \\
Penicillium crustosum & MPA 1412 & Bruker database \\
Penicillium turbatum & DSM 2426 T & Bruker database \\
Penicillium roqueforti & DSM 1079 & Bruker database \\
Penicillium lanosum & UGB 411 & Bruker database \\
Penicillium expansum & DSM 1282 & Bruker database \\
Penicillium digitatum & LLH 1625664 & Bruker database \\
Penicillium italicum & DSM 2417 & Bruker database \\
Penicillium commune & MPA 1260 & Bruker database \\
Penicillium chrysogenum & MPA 1262 & Bruker database \\
Penicillium verrucosum & DSM 12639 & Bruker database \\
Penicillium olsonii & DSM 16515 & Bruker database \\
Penicillium brevicompactum & DSM 21173 & Bruker database \\
Penicillium rugulosum & DSM 19649 & Bruker database \\
Penicillium pseudostromaticum & DSM 2421 & Bruker database \\
Penicillium purpurogenum & DSM 21170 & Bruker database \\
Penicillium funiculosum & MPA 1271 & \\
Penicillium marneffei & ATCC $182244^{\top}$ & Mold form (20 strains) \\
Penicillium marneffei & Yeast form (20 strains) & \\
\hline & & \\
& & \\
& & \\
& &
\end{tabular}

immunocompromised patients, identified as other Penicillium species with low scores using the Bruker Filamentous Fungi Library 1.0, should raise the suspicion of P. marneffei. When the combined database is expanded with spectra from $21 \mathrm{P}$. marneffei strains grown in mold, yeast or both phases, all the remaining 39 strains can be correctly identified to the species level with score $>2.0$. We also showed that different strains of $P$. marneffei displayed very similar MALDI-TOF MS profiles. As a result, no obvious clustering can be observed between the mold and yeast forms of the same strain in the dendrogram (Fig. 2). This suggests that either mold or yeast cultures of $P$. marneffei can be accurately identified by MALDI-TOF MS, which may potentially replace the tedious culture and morphological examination, and allows timely diagnosis and treatment of penicilliosis.

The most critical bottleneck to improve the performance of MALDI-TOF MS for identifying the less 


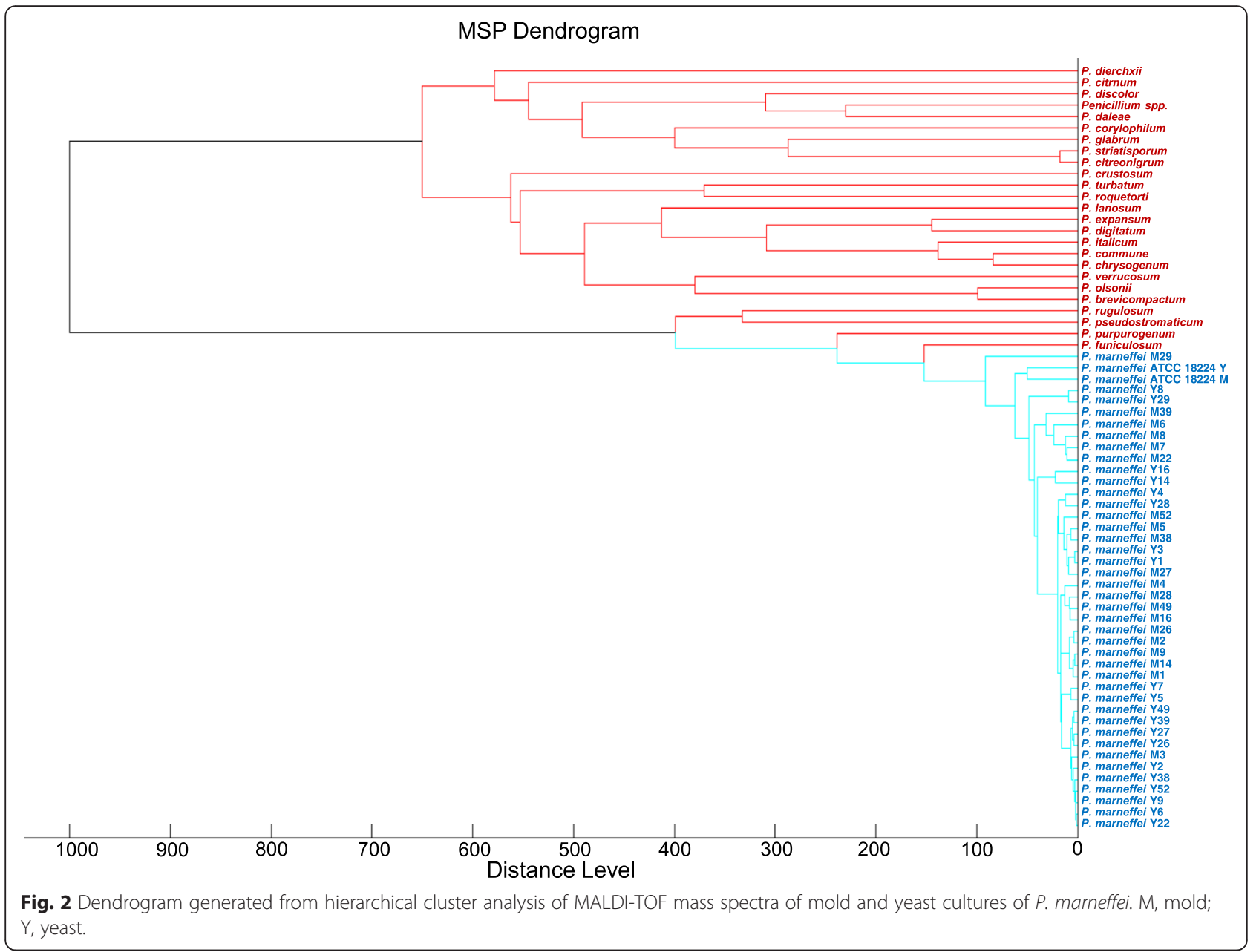

commonly encountered pathogens mainly lies in the composition of the database. Despite the scarcity of reports on the used of MALDI-TOF MS for identifying dimorphic fungi, a recent study tested various clinically encountered molds including $P$. marneffei [45]. The MALDI-TOF Bruker Biotyper system was unable to identify all 28 P. marneffei isolates. However, using a newly created database with one $P$. marneffei strain, $85.7 \%$ of their P. marneffei strains can be accurately identified with score $\geq 2.0$. This is in line with the present results, and suggests that adding more spectra for $P$. marneffei should further improve the success rate of identification as shown in this study. In this respect, we are also willing to share our $P$. marneffei MALDI-TOF MS spectra with interested laboratories to assist the early diagnosis of penicilliosis. The suboptimal database is also likely the reason for the failure in identifying the two other Penicillium and two Talaromyces species. Although P. brevi-compactum and P. chrysogenum can be correctly identified to their respective species as the best matches using databases with or without spectra from P. marneffei, the scores were below the cutoff (2.0) required for species identification. According to the interpretative criteria for Bruker database, the present $P$. brevi-compactum strain would be identified to the genus level as Penicillium species (scores 1.714 to 1.736) and the $P$. chrysogenum strain would be unidentified (scores 1.689). The low scores may be explained by the limited number of spectra for the two species (five spectra for each species) in the Bruker Filamentous Fungi Library, which is inadequate to accommodate intraspecies variability. On the other hand, T. aurantiacus and $T$. stipitatus would be unidentified according to the same criteria (scores for best matches 1.343-1.604). While $T$. aurantiacus showed the best match to $P$. purpurogenum or $P$. marneffei, surprisingly, T. stipitatus showed the best match to the distantly related bacterial species, $P$. putida or L. plantarum. This also reflects the lack of spectra for the genus Talaromyces and related spectra in the database. Penicillium and Talaromyces species other than P. marneffei are occasionally cultured from clinical specimens such as infected nails. Therefore, inclusion of more spectra from each of these species may improve the performance of MALDI-TOF 
MS for their identification, and help better understand their disease associations.

Although MALDI-TOF MS is proven to be useful for identification of various bacterial and fungal pathogens, commercial databases may not contain the less commonly encountered pathogens, newly discovered species or those pathogens limited to certain geographical areas. We have previously shown that MALDI-TOF MS is useful for identifying various "difficult-to-identify" bacterial pathogens, as well as Burkholderia pseudomallei which is also endemic in southeast Asia [24, 25]. However, the accurate identification of these less common pathogens often requires the expansion of exsiting databases using bacterial strains from the respective species. Since commonly encountered pathogens are often readily identified by phenotypic tests, it is anticipated that the major role of MALDI-TOF in clinical laboratories would be for the identification of the less common or "difficult-toidentify" pathogens. Therefore, further improvement of commercial databases is important to allow the use of MALDI-TOF MS for routine fungal identification. Genotypic methods, such as sequencing of internal transcribed spacer regions, still remain as the current gold standard for fungal identification, which can be partly explained by vast sequence data available in public databases such as GenBank. However, sequencing is still expensive and time-consuming to be routinely used in clinical laboratories. With improvement of commercial databases, MALDI-TOF MS may replace genotypic methods as a cost-saving, first-line identification system for the less common fungal pathogens. Compared to other MALDI-TOF MS systems such as Vitek MS which also used for identification of various bacteria and fungi, the Bruker system has the advantage of being more versatile and allowing database enlargements [46]. Therefore, the Bruker system should be considered by laboratories which foresee the need of expanding databases for identifying less common pathogens such as $P$. marneffei.

\section{Conclusions}

MALDI-TOF MS is useful for rapid identification of both yeast and mold cultures of P. marneffei. However, accurate identification to the species level requires expansion of the database using $P$. marneffei strains. In countries where $P$. marneffei is endemic, expansion of MALDI-TOF MS databases using different $P$. marneffe $i$ strains should be considered in clinical microbiology laboratories. This can be easily achieved using yeast cultures which are safer to handle than mold culture, with only tiny amount of fungal cells from a single colony subject to ethanol-formic acid extraction. The use of this low-cost, rapid and easy-to-perform state-of-the-art technology may help expedite laboratory diagnosis and treatment of penicilliosis.

\section{Ethics approval and consent to participate}

Not applicable

\section{Consent for publication \\ Not applicable}

\section{Availability of data and material}

We are willing to share the MALDI-TOF MS spectra of our $P$. marneffei strains with interested researchers and laboratories.

\section{Abbreviations}

ATCC: American type culture collection; HIV: human immunodeficiency virus; MALDI-TOF: Matrix-assisted laser desorption ionization time-of-flight mass spectrometry.

\section{Competing interests}

The authors declare that they have no competing interests.

\section{Authors' contributions}

SKPL, BSFT and PCYW coordinated the study. SKPL, CSKL and WNC performed data analysis. CSKL, AHYN and WNC performed experiments. SKPL, AKLW, DNCT, CWST, TLQ and PCYW collected fungal isolates. SKPL, CSKL, WNC and PCYW wrote the manuscript. All authors read and approved the final manuscript.

\section{Acknowledgements}

We thank Wai-Sing Chan, Ting-Yin Wong and Yu-Ting Tong for technical assistance.

\section{Funding}

This work is partly supported by the Health and Medical Research Fund (no. HKM-15-M07 [commissioned project]), Food and Health Bureau, Government of the Hong Kong Special Administrative Region, Hong Kong; the Shaw Foundation, Committee for Research and Conference Grant and University Development Fund, The University of Hong Kong. The funding bodies have no role in the design of the study and collection, analysis, and interpretation of data and in writing the manuscript.

\section{Author details}

${ }^{1}$ State Key Laboratory of Emerging Infectious Diseases, The University of Hong Kong, Hong Kong, China. ${ }^{2}$ Research Centre of Infection and Immunology, The University of Hong Kong, University Pathology Building, Queen Mary Hospital, Hong Kong, China. ${ }^{3}$ Carol Yu Centre for Infection, The University of Hong Kong, Hong Kong, China. ${ }^{4}$ Department of Microbiology, The University of Hong Kong, Hong Kong, China. ${ }^{5}$ Hong Kong Sanatorium Hospital, Hong Kong, China. ${ }^{6}$ Department of Pathology, Pamela Youde Nethersole Eastern Hospital, Hong Kong, China. ${ }^{7}$ Department of Pathology, Queen Elizabeth Hospital, Hong Kong, China. ${ }^{8}$ Department of Pathology, Kwong Wah Hospital, Hong Kong, China. ${ }^{9}$ Department of Pathology, Tuen Mun Hospital, Hong Kong, China.

Received: 23 April 2015 Accepted: 2 March 2016

Published online: 10 March 2016

\section{References}

1. Hsueh PR, Teng LJ, Hung CC, Hsu JH, Yang PC, Ho SW, et al. Molecular evidence for strain dissemination of Penicillium marneffei: an emerging pathogen in Taiwan. J Infect Dis. 2000;181:1706-12.

2. Supparatpinyo K, Khamwan C, Baosoung V, Nelson KE, Sirisanthana T. Disseminated Penicillium marneffei infection in southeast Asia. Lancet. 1994; 344:110-3.

3. Huang YT, Hung CC, Liao CH, Sun HY, Chang SC, Chen YC. Detection of circulating galactomannan in serum samples for diagnosis of Penicillium marneffei infection and cryptococcosis among patients infected with human immunodeficiency virus. J Clin Microbiol. 2007:45:2858-62.

4. Yuen KY, Wong SS, Tsang DN, Chau PY. Serodiagnosis of Penicillium marneffei infection. Lancet. 1994;344:444-5. 
5. Chariyalertsak S, Vanittanakom P, Nelson KE, Sirisanthana T, Vanittanakom N. Rhizomys sumatrensis and Cannomys badius, new natural animal hosts of Penicillium marneffei. J Med Vet Mycol. 1996;34:105-10.

6. Deng ZL, Yun M, Ajello L. Human penicilliosis marneffei and its relation to the bamboo rat (Rhizomys pruinosus). J Med Vet Mycol. 1986;24:383-9.

7. Deng ZL, Connor DH. Progressive disseminated penicilliosis caused by Penicillium marneffei. Report of eight cases and differentiation of the causative organism from Histoplasma capsulatum. Am J Clin Pathol. 1985;84:323-7.

8. Low K, Lee SS. The pattern of AIDS Reporting and the implications on HIV surveillance. Public Health Epidemiol Bull. 2002;11:41-9.

9. Wong $\mathrm{KH}$, Lee SS. Comparing the first and second hundred AIDS cases in Hong Kong. Singapore Med J. 1998;39:236-40.

10. Lo CY, Chan DT, Yuen KY, Li FK, Cheng KP. Penicillium marneffei infection in a patient with SLE. Lupus. 1995:4:229-31.

11. Wang JL, Hung CC, Chang SC, Chueh SC, La MK. Disseminated Penicillium marneffei infection in a renal-transplant recipient successfully treated with liposomal amphotericin B. Transplantation. 2003;76:1136-7.

12. Wong SSY, Woo PCY, Yuen KY. Candida tropicalis and Penicillium marneffei mixed fungaemia in a patient with Waldenstrom's macroglobulinaemia.

Eur J Clin Microbiol Infect Dis. 2001;20:132-5.

13. Woo PC, Lau SK, Lau CC, Chong KT, Hui WT, Wong SS, et al. Penicillium marneffei fungaemia in an allogeneic bone marrow transplant recipient. Bone Marrow Transplant. 2005;35:831-3.

14. Sekhon AS, Stein L, Garg AK, Black WA, Glezos JD, Wong C. Pulmonary penicillosis marneffei: report of the first imported case in Canada. Mycopathologia. 1994;128:3-7.

15. Vanittanakom N, Jr Cooper CR, Fisher MC, Sirisanthana T. Penicillium marneffei infection and recent advances in the epidemiology and molecular biology aspects. Clin Microbiol Rev. 2006;19:95-110.

16. Woo PC, Tam EW, Chong KT, Cai JJ, Tung ET, Ngan AH, et al. High diversity of polyketide synthase genes and the melanin biosynthesis gene cluster in Penicillium marneffei. FEBS J. 2010;277:3750-8.

17. Woo PC, Lam CW, Tam EW, Leung CK, Wong SS, Lau SK, et al. First discovery of two polyketide synthase genes for mitorubrinic Acid and mitorubrinol yellow pigment biosynthesis and implications in virulence of Penicillium marneffei. PLoS Negl Trop Dis. 2012;6:e1871.

18. Lau SKP, Chow WN, Wong AYP, Yeung JM, Bao J, Zhang N, et al. Identification of microRNA-like RNAs in mycelial and yeast phases of the thermal dimorphic fungus Penicillium marneffei. PLoS Negl Trop Dis. 2013;8: e2398.

19. Lau SKP, Tse H, Chan JSY, Zhou AC, Curreem SO, Lau CC, et al. Proteome profiling of the dimorphic fungus Penicillium marneffei extracellular proteins and identification of glyceraldehyde-3-phosphate dehydrogenase as an important adhesion factor for conidial attachment. FEBS J. 2013;280:6613-26.

20. Frisvad JC, Yilmaz N, Thrane U, Rasmussen KB, Houbraken J, Samson RA. Talaromyces atroroseus, a new species efficiently producing industrially relevant red pigments. PLoS ONE. 2013;8:e84102.

21. Bizzini A, Greub G. Matrix-assisted laser desorption ionization time-of-flight mass spectrometry, a revolution in clinical microbial identification. Clin Microbiol Infect. 2010;16:1614-9.

22. Seng $P$, Drancourt $M$, Gouriet $F$, La Scola B, Fournier PE, Rolain JM, et al. Ongoing revolution in bacteriology: routine identification of bacteria by matrix-assisted laser desorption ionization time-of-flight mass spectrometry. Clin Infect Dis. 2009;49:543-51.

23. Stevenson LG, Drake SK, Shea YR, Zelazny AM, Murray PR. Evaluation of matrix-assisted laser desorption ionization-time of flight mass spectrometry for identification of clinically important yeast species. J Clin Microbiol. 2010; 48:3482-6.

24. Lau SK, Tang BS, Teng JL, Chan TM, Curreem SO, Fan RY, et al. Matrixassisted laser desorption ionisation time-of-flight mass spectrometry for identification of clinically significant bacteria that are difficult to identify in clinical laboratories. J Clin Pathol. 2014:67:361-6.

25. Lau SK, Tang BS, Curreem SO, Chan TM, Martelli P, Tse CW, et al. Matrixassisted laser desorption ionization-time of flight mass spectrometry for rapid identification of Burkholderia pseudomallei: importance of expanding databases with pathogens endemic to different localities. J Clin Microbiol. 2012;50:3142-3.

26. Karger A, Stock R, Ziller M, Elschner MC, Bettin B, Melzer F, et al. Rapid identification of Burkholderia mallei and Burkholderia pseudomallei by intact cell Matrix-assisted Laser Desorption/lonisation mass spectrometric typing. BMC Microbiol. 2012:12:229.

27. De Carolis E, Posteraro B, Lass-Flörl C, Vella A, Florio AR, Torelli R, et al. Species identification of Aspergillus, Fusarium and Mucorales with direct surface analysis by matrix-assisted laser desorption ionization time-of-flight mass spectrometry. Clin Microbiol Infect. 2012;18:475-84.

28. Alanio A, Beretti JL, Dauphin B, Mellado E, Quesne G, Lacroix C, et al. Matrix-assisted laser desorption ionization time-of-flight mass spectrometry for fast and accurate identification of clinically relevant Aspergillus species. Clin Microbiol Infect. 2011;17:750-5.

29. Posteraro B, Vella A, Cogliati M, De Carolis E, Florio AR, Posteraro P, et al. Matrix-assisted laser desorption ionization-time of flight mass spectrometrybased method for discrimination between molecular types of Cryptococcus neoformans and Cryptococcus gattii. J Clin Microbiol. 2012;50:2472-6.

30. Chen JH, Yam WC, Ngan AH, Fung AM, Woo WL, Yan MK, et al. Advantages of using matrix-assisted laser desorption ionization-time of flight mass spectrometry as a rapid diagnostic tool for identification of yeasts and mycobacteria in the clinical microbiological laboratory. J Clin Microbiol. 2013;51:3981-7.

31. Ling H, Yuan Z, Shen J, Wang Z, Xu Y. Accuracy of matrix-assisted laser desorption ionization-time of flight mass spectrometry for identification of clinical pathogenic fungi: a meta-analysis. J Clin Microbiol. 2014;52: 2573-82

32. Won EJ, Shin JH, Lee K, Kim MN, Lee HS, Park YJ, et al. Accuracy of specieslevel identification of yeast isolates from blood cultures from 10 university hospitals in South Korea by use of the matrix-assisted laser desorption ionization-time of flight mass spectrometry-based Vitek MS system. J Clin Microbiol. 2013:51:3063-5.

33. Kolecka A, Khayhan K, Groenewald M, Theelen B, Arabatzis M, Velegraki A, et al. Identification of medically relevant species of arthroconidial yeasts by use of matrix-assisted laser desorption ionization-time of flight mass spectrometry. J Clin Microbiol. 2013;51:2491-500.

34. Alshawa K, Beretti JL, Lacroix C, Feuilhade M, Dauphin B, Quesne G, et al. Successful identification of clinical dermatophyte and Neoscytalidium species by matrix-assisted laser desorption ionization-time of flight mass spectrometry. J Clin Microbiol. 2012;50:2277-81.

35. Theel ES, Hall L, Mandrekar J, Wengenack NL. Dermatophyte identification using matrix-assisted laser desorption ionization-time of flight mass spectrometry. J Clin Microbiol. 2011;49:4067-71.

36. Normand AC, Cassagne C, Ranque S, L'ollivier C, Fourquet P, Roesems S, et al. Assessment of various parameters to improve MALDI-TOF MS reference spectra libraries constructed for the routine identification of filamentous fungi. BMC Microbiol. 2013;13:76.

37. Marinach C, Alanio A, Palous M, Kwasek S, Fekkar A, Brossas JY, et al. MALDI-TOF MS-based drug susceptibility testing of pathogens: the example of Candida albicans and fluconazole. Proteomics. 2009;9:4627-31.

38. Vella A, De Carolis E, Vaccaro L, Posteraro P, Perlin DS, Kostrzewa M, et al. Rapid antifungal susceptibility testing by matrix-assisted laser desorption ionization-time of flight mass spectrometry analysis. J Clin Microbiol. 2013; 51:2964-9.

39. Segretain G. Penicillium marneffei n.sp., agent of a mycosis of the reticuloendothelial system. Mycopathologia. 1959;11:327-53.

40. Woo PC, Lau CC, Chong KT, Tse H, Tsang DN, Lee RA, et al. MP1 homologue-based multilocus sequence system for typing the pathogenic fungus Penicillium marneffei: a novel approach using lineage-specific genes. J Clin Microbiol. 2007;45:3647-54.

41. Woo PC, Zhen H, Cai JJ, Yu J, Lau SK, Wang J, et al. The mitochondrial genome of the thermal dimorphic fungus Penicillium marneffei is more closely related to those of molds than yeasts. FEBS Lett. 2003; 555:469-77.

42. Eigner U, Holfelder M, Oberdorfer K, Betz-Wild U, Bertsch D, Fahr AM. Performance of a matrix-assisted laser desorption ionization-time-of-flight mass spectrometry system for the identification of bacterial isolates in the clinical routine laboratory. Clin Lab. 2009;55:289-96.

43. Tsang CC, Chan JF, Ip PP, Ngan AH, Chen JH, Lau SK, et al. Subcutaneous phaeohyphomycotic nodule due to Phialemoniopsis hongkongensis sp. nov. J Clin Microbiol. 2014;52:3280-9.

44. Lau AF, Drake SK, Calhoun LB, Henderson CM, Zelazny AM. Development of a clinically comprehensive database and a simple procedure for identification of molds from solid media by matrix-assisted laser desorption ionization-time of flight mass spectrometry. J Clin Microbiol. 2013;51:828-34. 
45. Chen YS, Liu YH, Teng SH, Liao CH, Hung CC, Sheng WH, et al. Evaluation of the matrix-assisted laser desorption/ionization time-of-flight mass spectrometry Bruker Biotyper for identification of Penicillium marneffei, Paecilomyces species, Fusarium solani, Rhizopus species, and Pseudallescheria boydii. Front Microbiol. 2015;6:679.

46. Mancini N, De Carolis E, Infurnari L, Vella A, Clementi N, Vaccaro L, et al. Comparative evaluation of the Bruker Biotyper and Vitek MS matrix-assisted laser desorption ionization-time of flight (MALDI-TOF) mass spectrometry systems for identification of yeasts of medical importance. J Clin Microbiol. 2013:51:2453-7.

Submit your next manuscript to BioMed Central and we will help you at every step:

- We accept pre-submission inquiries

- Our selector tool helps you to find the most relevant journal

- We provide round the clock customer support

- Convenient online submission

- Thorough peer review

- Inclusion in PubMed and all major indexing services

- Maximum visibility for your research

Submit your manuscript at www.biomedcentral.com/submit 